\title{
Miedo a la evaluación negativa, autoestima y presión psicológica: Efectos sobre el rendimiento deportivo en adolescentes
}

\author{
Fear of negative evaluation, self-esteem and choking under \\ pressure: Effects on sport performance in adolescents
}

\section{Medo da avaliação negativa, auto-estima e pressão psicológica: efeitos sobre o rendimento desportivo em adolescentes}

\author{
José Molina, Paloma Chorot, Rosa M. Valiente y Bonifacio Sandín
}

Facultad de Psicología, Universidad Nacional de Educación a Distancia, Madrid (España)

\begin{abstract}
Resumen: El objetivo del presente estudio consistió en examinar el miedo a la evaluación negativa y la autoestima como posibles factores moduladores del choking (caída del rendimiento deportivo asociado a la presión psicológica). Participaron 100 estudiantes de secundaria (el 56\% eran mujeres), los cuales cumplimentaron la Brief Fear of Negative Evaluation (BFNE) y la Self-Esteem Scale (SES) antes de participar en un set de bádminton en condiciones de baja y alta presión. Los datos basados en ANOVAs apoyan la hipótesis de que el miedo a la evaluación negativa actúa como modulador del deterioro del rendimiento deportivo. Los participantes con elevado nivel de miedo a la evaluación negativa experimentaron una caída significativa en el rendimiento deportivo durante la condición de alta presión; el efecto de la autoestima se asoció, en general, a mayor rendimiento deportivo. El presente estudio amplía la literatura sobre el choking en el ámbito del deporte, proporcionando evidencia sobre la implicación del miedo a la evaluación negativa y la autoestima en el campo de la psicología de la actividad física y el deporte. Palabras clave: Choking; rendimiento deportivo; miedo a la evaluación negativa; autoestima; deporte; actividad física; adolescentes.

Abstract: The aim of the present study was to examine the fear of negative evaluation and self-esteem as moderators of choking in sport. One hundred high school students (56\% were females) completed the Brief Fear of Negative Evaluation (BFNE) and the Self-Esteem Scale (SES) prior to completing a badminton set in low-pressure and high-pressure conditions. Data based on a series of ANOVAs supported the hypothesis that fear of negative evaluation acts as a moderator of the choking effect under psycho-
\end{abstract}

logical pressure. Participants with high fear of negative evaluation showed a significant drop on sport performance from low- to high-pressure phases. In general, high-self-esteem was associated to high levels of performance. The present study extends the choking literature, including the constructs of fear of negative evaluation and self-esteem as possible relevant variables implicated in the field of sport performance.

Keywords: Choking; fear of negative evaluation; self-esteem; sport; physical activity; adolescents.

Resumo: O objectivo do presente estudo consistiu em examinar o medo da avaliação negativa e a auto-estima como possíveis factores modeladores do choking (quebra de rendimento desportivo associada à pressão psicológica). Participaram 100 estudantes do ensino secundário (56\% eram mulheres), os quais completaram a BriefFear of Negative Evaluation (BFNE) e a Self-Esteem Scale (SES) antes de participarem num set de badminton em condições de baixa e elevada pressão. Os dados baseados em ANOVAs apoiam a hipótese de que o medo da avaliaçấo negativa actua como moderador do deterioro do rendimento desportivo. Os participantes com elevado nível de medo da avaliaçâo negativa experienciaram uma quebra significativa no rendimento desportivo durante a condição de elevada pressão; o efeito da auto-estima associou-se, em geral, a um superior rendimento desportivo. O presente estudo amplia a literatura sobre o choking no âmbito do desporto, proporcionando evidência sobre a implicação do medo da avaliação negativa e da auto-estima no campo da psicologia da actividade física e do desporto. Palavras-chave: Choking; rendimento desportivo; medo da avaliação negativa; auto-estima; desporto; actividade física; adolescentes.

\section{Introducción}

El rendimiento deportivo es un factor central en gran parte de las situaciones en que se llevan a cabo actividades deportivas. Así, tanto el deporte de alto rendimiento como, en muchas ocasiones, el deporte a nivel educativo, son modalidades donde la efectividad por parte del sujeto se hace indispensable si se quiere obtener el éxito y logro deportivo. Se ha indicado que el rendimiento deportivo puede estar determinado

\footnotetext{
Dirección para correspondencia [Correspodence address]: Bonifacio Sandín, Universidad Nacional de Educación a Distancia, Facultad de psicología, Juan del Rosal 10, 28040 Madrid (España). E-mail: bsandin@psi.uned.es
}

por diversas variables psicológicas, tales como la motivación, la atención, el estrés, la ansiedad, la autoconfianza, los estados de ánimo, la autoestima, o el miedo a la evaluación social (Auweele, De-Cuyper, Van-Mele, y Rzewnicky, 1993; Buceta, 1996; Coudevylle, Gernigon y Martin, 2011; Hammermeister y Burton, 2001; Mesagno, Harvey y Janelle, 2012). Por ello, resulta de gran relevancia el conocimiento del papel que puedan desempeñar las variables psicológicas en orden a optimizar el rendimiento en el deporte, sea este de tipo competitivo o de tipo educativo.

Las situaciones en las que se estudia el rendimiento de- 
portivo suelen implicar un incremento significativo de los estados de activación, ansiedad y estrés del deportista. Habitualmente en este tipo de situaciones, donde se busca el rendimiento como vía para superar al rival o a sí mismo, suele aparecer el fenómeno de "presión psicológica" sobre el deportista, el cual puede llegar a mermar sus facultades de cara a la competición y el rendimiento. Este concepto es denominado en la literatura científica como "choking under pressure" o "asfixia bajo presión", y aunque es un término muy utilizado, una definición unitaria sobre el mismo aún no ha sido definitivamente delimitada (Hill, Hanton, Mattews, Fleming, 2010). Aunque en el campo de la psicología del deporte se han utilizado, de forma más o menos indiferenciada, los conceptos de activación (o arousal), estrés y ansiedad, cuando se trata de competiciones deportivas, donde se busca obtener un rendimiento óptimo, se ha propuesto como más relevante el concepto de "presión psicológica deportiva" que, aunque puede incluir aspectos de los tres conceptos anteriores, puede aplicarse de forma más específica al ámbito deportivo.

En este sentido, Baumeister (1984) definió la asfixia bajo presión como una expresión metafórica usada para describir la ocurrencia de una reducción del rendimiento deportivo a pesar de los esfuerzos del individuo por conseguir un rendimiento superior. La presión se ha definido como un factor o combinación de factores que incrementan la demanda de la situación (mayor importancia por conseguir un elevado rendimiento). El choking se refiere a la reducción del rendimiento bajo las situaciones de presión. El "ahogamiento" o asfixia bajo presión se ha asociado generalmente al ámbito de la competición deportiva, ya que la competición constituye en sí misma una forma de presión, la cual suele asociarse a incrementos significativos de los niveles de activación (Baumeister, 1984).

Posteriormente, Hall (2002) redefinió el choking como una elevación en los niveles de ansiedad y activación bajo presión percibida, que conduce a un deterioro crítico en la ejecución habitual del rendimiento. Este concepto de presión psicológica en el deportista, choking o asfixia bajo presión (Hill et al., 2010), ha sido interpretado como un grave deterioro en la ejecución de los procesos habituales, como resultado de una elevación en la ansiedad bajo la presión psicológica percibida, lo cual puede llevar a reducciones significativas en el desempeńo (Mesagno, Marchant y Morris, 2008).

Aunque en general la psicología del deporte ha valorado la relevancia de conceptos clásicos de la ansiedad, tales como las dimensiones cognitiva y somática (Jones, 1995; Woodman y Hardy, 2001; Sandín, 2010), existen otros constructos psicológicos relacionados con la ansiedad que, aunque menos investigados, podrían desempeńar un papel particularmente relevante. En este sentido, parece desempeñar un papel especialmente relevante la denominada sensibilidad a la ansiedad (miedo a los síntomas de ansiedad), variable de diferencias individuales que posee la propiedad de motivar o amplificar cualquier tipo de miedo (Sandín, Chorot, Santed y Valiente, 2002; Sandín, Valiente, Chorot y Santed, 2005). Aplicado al ámbito deportivo, se ha referido que la tendencia a evitar las sensaciones corporales asociadas al ejercicio físico podría incrementar el miedo a los síntomas físicos de la ansiedad mediante un proceso de incubación (Gimeno y Ezquerro, 2006; Sandín y Chorot, 1989). La sensibilidad a la ansiedad, por tanto, podría interferir de forma significativa en el rendimiento deportivo en condiciones de estrés elevado, debido al incremento de la sintomatología asociada a la ansiedad y a las posibles tendencias hacia la evitación interoceptiva (Sandín, 2005).

En un estudio reciente, Molina, Sandín y Chorot (2014) examinaron el posible papel de la sensibilidad a la ansiedad sobre el rendimiento deportivo (un juego de bádminton) en condiciones de alta y baja presión psicológica en una muestra de adolescentes. Partiendo de que la elevada presión psicológica tiende a reducir el rendimiento deportivo, Molina et al. investigaron el posible efecto modulador de la sensibilidad a la ansiedad sobre el efecto que induce la presión psicológica en el rendimiento deportivo. Encontraron un efecto modulador de esta variable, la cual incrementaba significativamente el efecto negativo sobre el rendimiento inducido por la condición de alta presión psicológica. Estos resultados confirman la hipótesis de que el temor a los síntomas de ansiedad (p.ej., los síntomas físicos de ansiedad) puede interferir en el desempeño deportivo en condiciones de altos niveles de estrés, bien a través de procesos de evitación interoceptiva (Gimeno y Ezquerro, 2006; Sandín, 2005), de procesos de incubación de la ansiedad (Sandín y Chorot, 1989; Chorot y Sandín, 1993), y/o a través de procesos de distracción o interferencia relacionados con la ansiedad (Hill y Shaw, 2013).

El miedo a la evaluación negativa (i.e., sensibilidad a los síntomas de evaluación social) es una variable estrechamente relacionada con la sensibilidad a la ansiedad. Fue definida por Watson y Friend (1969) como aprensión ante la evaluación de los demás, la angustia a las evaluaciones negativas, la evitación de las situaciones de evaluación, y las expectativas de que otras personas evalúen negativamente a uno mismo. La predisposición a experimentar miedo a la evaluación negativa tiene al menos dos implicaciones, una de tipo subjetivo ("experiencia subjetiva" de ansiedad) y otra de tipo comportamental ("consecuencias conductuales") (Leary (1983; Sandín, Chorot, Valiente, Santed y Sánchez-Arribas, 1999). Leary (1992) trasladó estos conceptos al ámbito de la psicología deportiva y conceptualizó la ansiedad competitiva como una clase específica de ansiedad social, defendiendo que la ansiedad competitiva se relaciona con las implicaciones de auto-representación de la competición. Basándonos en la conceptualización que hacen estos autores, la predisposición al miedo a la evaluación negativa debería amplificar la ansiedad de com- 
petición en situaciones de alta tensión deportiva. Así mismo, de acuerdo con los componentes asociados a la evaluación negativa (Leary, 1983; Sandín et al., 1999), la tendencia a la evaluación negativa debería interferir con el rendimiento deportivo en situaciones de alta presión deportiva (choking).

Aunque recientemente Mesagno et al. (2012) proporcionaron evidencia preliminar a favor de esta hipótesis, apenas se ha investigado la influencia que tiene el miedo a la evaluación negativa sobre el rendimiento deportivo. Estos autores, basándose en una muestra de jugadores de baloncesto, encontraron que la elevada predisposición al miedo a la evaluación negativa (grupo de alto miedo) redujo el rendimiento durante la condición de alta presión psicológica, mientras que el grupo de bajo miedo a la evaluación negativa exhibió niveles similares de rendimiento en ambas condiciones de presión psicológica (presión alta vs. presión baja). El temor a la evaluación parece interferir en el rendimiento deportivo, aunque únicamente cuando este se lleva a cabo en situaciones de alta presión psicológica, actuando por tanto como modulador del efecto de la presión psicológica.

La autoestima es una variable de personalidad referida al grado con que una persona se valora a sí misma (Rosenberg, 1965), habiéndose relacionado de forma positiva con la salud física (Montero, Rueda y Bermúdez, 2012) y psicológica (Sandín, Chorot, Lostao y Valiente, 2012). Se ha sugerido, así mismo, que la autoestima se asocia positivamente al bienestar mental en los atletas y deportistas por su relación con características psicológicas positivas (resiliencia, etc.) y emocionales (miedos, ansiedad, depresión, etc.), así como también con la evaluación de las situaciones estresantes (Baumeister, 1993; Montero et al., 2012; Sandín et al., 2012). Aunque existe amplia evidencia sobre la influencia favorable de la autoestima en el rendimiento (p.ej., el rendimiento académico; Aryana, 2010), llama la atención que esta variable apenas se haya investigado en relación con el rendimiento deportivo. Adie, Duda, y Ntoumanis (2008), en su estudio con participantes deportistas, encontraron que las personas con niveles bajos en autoestima tendían a percibir la competición deportiva de una forma más amenazante, mientras que las personas con niveles altos en autoestima percibían la competición como una actividad más exigente, lo que facilitaría la mejora del rendimiento deportivo.

El objetivo del presente estudio ha consistido en extender nuestros hallazgos previos sobre la relación entre la sensibilidad a la ansiedad y el rendimiento deportivo centrándonos en el papel de la sensibilidad a la evaluación negativa (o miedo a la evaluación negativa) y la autoestima. Partiendo de la evidencia de la literatura que hemos comentado atrás, en la presente investigación formulamos una primera hipótesis según la cual el miedo a la evaluación negativa debería modular el efecto de la presión psicológica, interfiriendo con el rendimiento en la condición de alta presión psicológica, y facilitando el rendimiento en la condición de baja presión; esta segunda parte de la hipótesis la basamos en que el miedo puede actuar como drive motivando y facilitando la conducta en condiciones de bajo estrés (Chorot y Sandín, 1993; Arent y Landers, 2003). Así mismo, en línea con la propuesta de Mesagno et al. (2012), hipotetizamos un incremento del rendimiento deportivo durante el periodo de alta presión deportiva en el grupo de participantes de bajo miedo (segunda hipótesis). Así mismo, sobre la base de esta evidencia preliminar, esperamos que la autoestima debería facilitar el rendimiento deportivo, especialmente en la situación de alta presión deportiva (tercera hipótesis). Dada la escasa evidencia que actualmente existe sobre el posible efecto de la autoestima sobre el rendimiento deportivo, entendemos que esta cuarta hipótesis tiene un carácter exploratorio.

\section{Método}

\section{Diseño}

Se aplicó un diseño de dos condiciones experimentales de medidas repetidas. Las condiciones experimentales consistían en una condición de baja presión deportiva y una condición de alta presión deportiva (el diseño incluye también una fase previa de familiarización). El procedimiento ha resultado ser efectivo para inducir situaciones de alta y baja presión psicológica en el contexto del deporte de bádminton (Molina et al., 2014). Las condiciones correspondían a partidos de bádminton de un juego. Todos los participantes participaron en ambas condiciones. (Véase la sección sobre el procedimiento).

\section{Participantes}

Se seleccionó una muestra de 100 alumnos del Instituto de Enseñanza Secundaria Mónico Sánchez (Piedrabuena, Ciudad Real), pertenecientes al curso académico 2011/2012. Los alumnos cursaban el nivel post-obligatorio de Bachillerato en cualquiera de las dos modalidades que oferta el centro educativo (Humanidades y Ciencias Sociales, Ciencias Naturales y Tecnológico). La muestra, por lo tanto, se basa en un muestreo no probabilístico compuesto por todos los alumnos de Bachillerato del citado centro educativo. La muestra estaba constituida por 44 varones y 56 mujeres, y estaba aproximadamente equiparada en edad entre varones y mujeres $(M=$ 16.1, $D T=0.55$; rango 15-17 años). Para la realización de los análisis estadísticos la muestra se dividió en dos grupos basándonos en las puntuaciones en la escala BFNE (bajo vs. alto) según que los participantes puntuaran por debajo $(n=$ $60)$ o por encima $(n=40)$ de la media, y en las puntuaciones de la escala SES (bajo vs. alto) según puntuaran por debajo $(n=48)$ o por encima $(n=52)$ de la media, respectivamente. Todos los participantes practicaban deporte con cierta regu- 
laridad; ninguno de ellos estaba federado en el deporte de bádminton.

\section{Instrumentos de evaluación}

Previo a la aplicación de las condiciones experimentales, los participantes cumplimentaron los siguientes cuestionarios de autoinforme de forma individual durante una sesión en el aula en horario de clase.

Brief Fear of Negative Evaluation (BFNE; Leary, 1983). Fue creada por Leary (1983) a partir de la Escala de Miedo a la Evaluación Negativa de Watson y Friend (1969). La BFNE está formada por 12 ítems que evalúan el grado en el que una persona teme ser evaluado negativamente por los demás. Tiene un formato de respuesta tipo Likert de 5 puntos, que puntúan entre 1 ("nada característico en mí") y 5 ("extremadamente característico en mí”) (cuatro de los items se puntúan de forma inversa). Utilizamos la versión española validada por Gallego, Botella, Quero, Bańos y García Palacios (2007). El coeficiente alfa de Cronbach obtenido por estas autoras es de .90 para la escala total. Gallego et al. también presentaron evidencia sobre validez convergente (correlaciones significativas con fobia social, depresión y ansiedad). El coeficiente alfa obtenido en el presente estudio fue de .82 .

Self-Esteem Scale (SES; Rosenberg, 1965). Desarrollada por Rosenberg para la evaluación de la autoestima en adolescentes, incluye diez ítems cuyos contenidos se centran en la valoración de las propias cualidades y aceptación de sí mismo. Consta de 10 items de autoinforme que el participante debe contestar según una escala Likert de 4 puntos, variando entre 1 ("muy en desacuerdo") y 4 ("muy de acuerdo"). La mitad de los items están formulados de forma inversa. Utilizamos la versión española validada por Sandín et al. (2012; Sandín, 2008). El coeficiente alfa obtenido en el presente estudio fue de .89 .

\section{Definición de las variables de rendimiento}

Durante las tres fases del experimento (fase de familiarización, fase de competición sin presión y fase de competición con presión) se evaluó la práctica deportiva de bádminton del participante a través de hojas de registro de observaciones, donde se registraron los valores individuales de cada uno de los participantes para las dos variables siguientes:

1. Errores no forzados (ENF) por el rival. Esta variable de rendimiento deportivo indica los errores que un jugador comete independientemente de la acción motriz del rival.

2. Puntos conseguidos (PC). Puntos que cada jugador consigue dentro de un set. Variable dependiente que resume las acciones motrices que conllevan a conseguir un tanto o punto.
Los registros fueron realizados por el profesor de la asignatura de Educación Física. Con objeto de probar la fiabilidad de las hojas de registro de las observaciones se procedió a analizar el grado de concordancia entre dos jueces (el profesor de la asignatura y otro profesor entrenado) que evaluaron 20 casos, seleccionados de manera aleatoria, obteniéndose un coeficiente kappa de Cohen de 0,98.

\section{Procedimiento}

El procedimiento se llevó a cabo en las cuatro fases siguientes:

Una primera fase (fase de aplicación de los cuestionarios de autoinforme) se utilizó para informar a los alumnos sobre el trabajo de investigación y obtener su consentimiento informado. Así mismo, durante esta fase se aplicaron las escalas de autoinforme BFNE y SES.

La segunda fase, previamente a la intervención, fue la fase de familiarización con la actividad deportiva del bádminton (i.e., situación de preparación y entrenamiento). En esta fase, que tuvo una duración de 15 sesiones de 55 minutos cada una, se inició la unidad de trabajo de bádminton, explicando las características del juego, el reglamento, evolución histórica, los golpes técnicos más relevantes, las situaciones tácticas más influyentes y la dinámica del juego. Posteriormente, se realizaron ejercicios prácticos y actividades de juego de bádminton con la finalidad que el alumno adquiriese una experiencia mínima, incluidas la ejecución de golpes y las tácticas. Al final de esta etapa se practicó en las situaciones de partido de modalidad individual y de dobles. El estudio (fases tercera y cuarta) se llevó a cabo en la modalidad de dobles.

La tercera fase correspondió a la fase de intervención sin presión (i.e., situación de competición de baja presión deportiva), las parejas participaron en una situación real de partido a un set de bádminton, fuera del horario lectivo de clase. Se explicó a los participantes que se trataba de una situación de competición sin evaluación (con la finalidad de reducir la presión deportiva), que serviría para preparar la situación posterior de competición con evaluación (i.e., fase con presión deportiva). Durante esta tercera fase los participantes jugaron un set de dobles de bádminton en condiciones de baja presión deportiva. Para conseguir este tipo de situación se eliminaron los elementos que habitualmente suelen incrementar el estrés deportivo y/o la ansiedad competitiva, tales como la asistencia de público, la percepción de ser grabado, y el que la práctica realizada influya en la calificación del alumno. Se realizó el registro durante el juego, cuantificándose los golpes ganadores y puntos conseguidos en el set.

Finalmente, la cuarta fase correspondió a la fase de intervención con presión (i.e., situación de competición de alta presión deportiva) y se llevó a cabo bajo las siguientes condiciones que supuestamente deberían incrementar el nivel de presión deportiva: (a) Las sesiones se llevaron a cabo durante las horas 
lectivas de clase de la asignatura de Educación Física. (b) El profesor grabó con una cámara fija con trípode el desempeño de cada pareja; los participantes sabían que estaban siendo grabados. (c) El profesor registraba el desempeño de los participantes, habiéndose informado que el resultado del partido influiría en la calificación de la evaluación de la asignatura de Educación Física. (d) Todos los alumnos de la clase observaron y registraron los desempeños de sus compañeros, con la finalidad de aumentar la presión psicológica sobre los participantes al verse estos últimos evaluados por sus compañeros. Además, a la pareja ganadora obtenían dos puntos (20\%) en la calificación final de la asignatura de Educación Física.

Este procedimiento de inducción experimental de presión (presión psicológica o deportiva) en el ámbito deportivo ha demostrado ser efectivo para manipular el nivel de presión (Molina et al., 2014). Estos autores demostraron que el rendimiento deportivo era significativamente menor en la condición de intervención de alta presión que en la condición de baja presión.

\section{Tratamiento estadístico de los datos}

Para poner a prueba las hipótesis formuladas en el presente estudio aplicamos dos diseńos de análisis de varianza (ANOVA) de $2 \times 2$. En el primero de estos diseños, la primera variable independiente estuvo constituida por 2 grupos de diferencias individuales basados en el nivel de miedo a la evaluación negativa (bajo vs. alto miedo a la evaluación negativa; $n$ 's $=60$ y 40 , respectivamente). La segunda variable independiente, también de dos niveles, estuvo determinada por las dos condiciones experimentales de presión deportiva (baja presión vs. alta presión). Mientras que el primer factor era un factor de grupo (factor de diferencias individuales), este segundo factor consistió en un factor de medias repetidas. El segundo diseño de ANOVA aplicado fue similar a este, excepto en que el factor de diferencias individuales estuvo determinado por los dos niveles de autoestima en lugar del miedo a la evaluación negativa (i.e., baja vs. alta autoestima; $n$ 's $=48$ y 52, respectivamente). Ambos diseños se aplicaron de forma separada para las variables dependientes "errores no forzados" y "puntos conseguidos".

\section{Resultados}

En la Tabla 1 indicamos los valores (medias y DTs) obtenidos para las 4 variables incluidas en el estudio (miedo a la evaluación negativa, autoestima, errores no forzados, y puntos conseguidos) en función del género. También se indican los valores de consistencia interna de las escalas BFNE y SES, los cuales resultaron ser elevados (en los dos casos fueron superiores a .80). Con objeto de examinar la diferencias según el género aplicamos un diseño de ANOVA de un factor (varón vs. mujer) para cada una de las cuatro variables. Como puede apreciarse, no encontramos diferencias significativas entre varones y mujeres en ninguna de las variables analizadas, excepto para la variable de errores no forzados en la condición de alta presión (en los subsiguientes análisis de varianza referidos a los errores no forzados se controló el efecto del género incluyendo esta variable como covariante).

Tabla 1. Medias y DTs para las variables de diferencias individuales (miedo a la evaluación negativa y autoestima) y de rendimiento deportivo, según género.

\begin{tabular}{|c|c|c|c|c|c|c|c|}
\hline & \multicolumn{2}{|c|}{ Varones } & \multicolumn{2}{|c|}{ Mujeres } & \multirow[b]{2}{*}{$F_{(1,98)}$} & \multirow[b]{2}{*}{ Eta $^{2}$ parcial } & \multirow[b]{2}{*}{$\alpha$} \\
\hline & Media & $D T$ & Media & $D T$ & & & \\
\hline BFNE & 31.34 & 9.62 & 32.96 & 10.13 & .66 & .01 & .92 \\
\hline SES & 31.36 & 5.66 & 32.77 & 5.02 & 1.72 & .01 & .86 \\
\hline Errores no forzados BP & 3.11 & 1.68 & 3.64 & 1.95 & 2.04 & .02 & \\
\hline Errores no forzados AP & 4.89 & 2.39 & 5.95 & 2.69 & $4.22^{*}$ & .04 & \\
\hline Puntos conseguidos BP & 8.23 & 3.11 & 8.13 & 3.13 & .03 & .00 & \\
\hline Puntos conseguidos AP & 7.05 & 3.54 & 6.18 & 3.35 & 1.57 & .02 & \\
\hline
\end{tabular}

Nota BFNE = Brief Fear of Negative Evaluation, $\mathrm{SES}=$ Self-Esteem Scale, BP = baja presión deportiva, AP = alta presión deportiva. ${ }^{*} \mathrm{p}<0.05$

\section{Efectos del miedo a la evaluación negativa sobre el rendimiento deportivo}

Con el fin de probar el posible efecto modulador del miedo a la evaluación negativa sobre el efecto de la presión deportiva aplicamos un diseño de análisis de varianza de $2 \times 2$ para cada una de las dos variables dependientes (errores no forzados $y$ puntos conseguidos). La primera variable independiente fue el miedo a la evaluación negativa y la segunda consistió en la condición experimental "presión deportiva" (baja presión vs. alta presión). Para ello, la primera variable se recodificó en valores bajo vs. alto según que los participantes puntuaran por debajo o por encima de la media. Los resultados de estos análisis aparecen en las Tablas 2 y 3. 
Tabla 2. Medias y DTs en errores no forzados en función de la condición experimental (baja vs. alta presión) y las variables de diferencias individuales (BFNE y SES) (nivel bajo vs. alto).

\begin{tabular}{|c|c|c|c|c|c|c|c|}
\hline & \multicolumn{2}{|c|}{ Baja Presión } & \multicolumn{2}{|c|}{ Alta presión } & \multirow[b]{3}{*}{$\mathrm{FPD}_{(1,98)}$} & \multirow[b]{3}{*}{$\mathrm{F} \mathrm{DI}_{(1,97)}$} & \multirow[b]{3}{*}{$\mathrm{F} P D \times \mathrm{DI}_{(1,97)}$} \\
\hline & Bajo & Alto & Bajo & Alto & & & \\
\hline & Media $(D T)$ & Media $(D T)$ & Media $(D T)$ & Media $(D T)$ & & & \\
\hline BFNE & $3.50(1.85)$ & $3.28(1.87)$ & $4.33(1.74)$ & $7.20(2.75)$ & $172.28^{* * *}$ & $11.91^{* *}$ & $71.28^{* * *}$ \\
\hline SES & $2.69(1.52)$ & $4.08(1.89)$ & $5.23(2.79)$ & $5.71(2.42)$ & $82.37^{* * *}$ & $5.58^{*}$ & $4.29^{*}$ \\
\hline
\end{tabular}

Nota. BFNE = Brief Fear of Negative Evaluation, SES = Self-Esteem Scale, F PD = valor del estadístico F para el factor de presión deportiva, F DI = valor del estadístico F para el factor de diferencias individuales, F PD x DI = valor del estadístico F para la interacción entre los factores de presión deportiva y diferencias individuales. ${ }^{*} \mathrm{p}<0.05 ;{ }^{* *} \mathrm{p}<0.01 ;^{* * *} \mathrm{p}<0.001$.

En la Tabla 2 indicamos los datos relativos a la variable dependiente errores no forzados. Como puede apreciarse, en general los errores se asocian en mayor medida a la condición de alta presión que a la de baja presión. El efecto del miedo a la evaluación negativa resultó ser estadísticamente significativo $(p<.01)$. Es importante seńalar que también fue significativa la interacción entre la condición experimental y el miedo a la evaluación negativa $(p<.001)$.

Con objeto de examinar de forma más específica esta interacción, indicamos en la Figura 1 los valores medios en errores no forzados en función de los niveles de ambos factores. Tal y como puede apreciarse en la figura, la mayor presión deportiva (condición de alta presión) incrementa el número de errores en ambos grupos (i.e., participantes altos vs. bajos en miedo a la evaluación negativa), si bien este incremento es mucho mayor en el grupo de miedo a la evaluación negativa elevado. Es decir, el miedo a la evaluación negativa tiende a modular el efecto de deterioro del rendimiento que induce la presión deportiva, facilitando la ocurrencia de errores no forzados. También merece resaltar, no obstante, que la condición de presión deportiva alta se asocia a un ligero incremento de los errores incluso en los sujetos con bajo nivel de miedo (aunque de forma mucho menos marcada).

Figura 1. Representación gráfica de la interacción entre el miedo a la evaluación negativa y la presión deportiva, para la variable dependiente errores no forzados. BFNE $=$ Brief Fear of Negative Evaluation

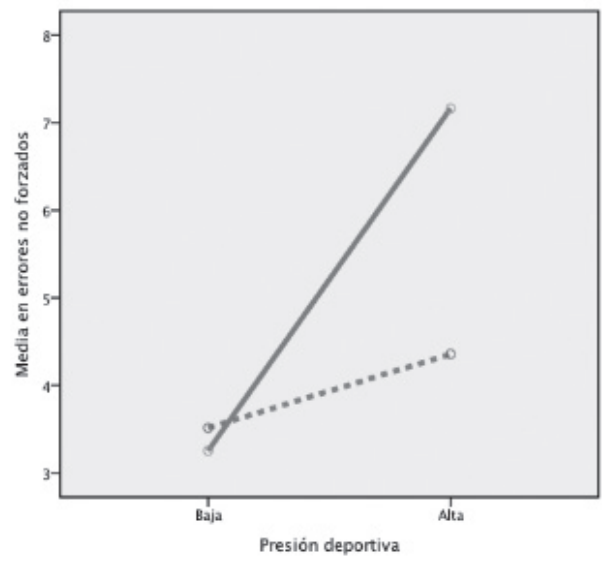

Miedo a la evaluación
negativa - - BFNE baja

Tabla 3. Medias y DTs en puntos conseguidos en función de la condición experimental (baja vs. alta presión) y las variables de diferencias individuales (BFNE y SES) (nivel bajo vs. alto).

\begin{tabular}{|c|c|c|c|c|c|c|c|}
\hline & \multicolumn{2}{|c|}{ Baja Presión } & \multicolumn{2}{|c|}{ Alta presión } & \multirow[b]{3}{*}{$\mathrm{F} \mathrm{PD}_{(1,98)}$} & \multirow[b]{3}{*}{$\mathrm{F} \mathrm{DI}_{(1,97)}$} & \multirow[b]{3}{*}{$F P D \times D_{(1,97)}$} \\
\hline & Bajo & Alto & Bajo & Alto & & & \\
\hline & Media (DT) & Media (DT) & Media (DT) & Media (DT) & & & \\
\hline BFNE & $7.73(3.33)$ & $8.82(2.63)$ & $7.77(2.91)$ & $4.75(3.42)$ & $74.07^{* * *}$ & 2.51 & $75.09^{* * *}$ \\
\hline SES & $7.44(3.10)$ & $8.85(2.98)$ & $5.52(3.28)$ & $7.52(3.34)$ & $28.12^{* * *}$ & $9.81^{* *}$ & 1.14 \\
\hline
\end{tabular}

Nota . BFNE = Brief Fear of Negative Evaluation, SES = Self-Esteem Scale, F PD = valor del estadístico F para el factor de presión deportiva, F DI = valor del estadístico F para el factor de diferencias individuales, F PD x DI = valor del estadístico F para la interacción entre los factores de presión deportiva y diferencias individuales. ${ }^{* *} \mathrm{p}<0.01{ }^{* * *} \mathrm{p}<0.001$.

En la Tabla 3 indicamos los datos relativos a la variable dependiente puntos conseguidos. Puede observarse que el patrón de rendimiento es diferente en función de ambas variables independientes (condición experimental y niveles de miedo). Así, mientras que el rendimiento en el grupo de bajo miedo es muy similar en ambas condiciones experimentales, el rendimiento del grupo de alto miedo se incrementa ligeramente en la condición de baja presión y cae de forma muy marcada en la condición de alta presión. Aunque el efecto global del miedo a la evaluación negativa no resultó estadísticamente significativo, sí lo fue la interacción de este factor con la condición experimental $(p<.001)$ (véase la Tabla 3). 
Figura 2. Representación gráfica de la interacción entre el miedo a la evaluación negativa y la presión deportiva, para la variable dependiente puntos conseguidos. BFNE = Brief Fear of Negative Evaluation.
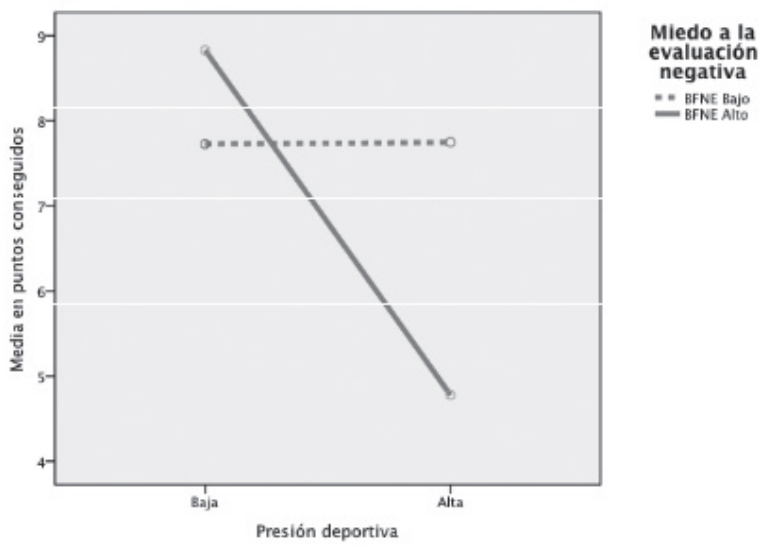

Esta interacción denota la existencia de un efecto modulador del miedo a la evaluación negativa sobre el efecto que induce la condición experimental sobre el rendimiento (puntos conseguidos) (véase la Figura 2). Como puede apreciarse en la figura, el miedo a la evaluación negativa parece modular el efecto de la condición experimental de un modo dual: por una parte deteriora marcadamente el rendimiento deportivo en condiciones de alta presión deportiva y, por otra, tiende a facilitar el rendimiento si la condición es de baja presión. En cualquier caso, el efecto negativo parece ser mucho más marcado que el efecto positivo sobre el rendimiento.

\section{Efectos de la autoestima sobre el rendimiento deportivo}

El análisis de los posibles efectos de la autoestima sobre el rendimiento deportivo se llevó a cabo siguiendo un procedimiento similar al que hemos indicado en relación con el miedo a la evaluación negativa (véase arriba). Los resultados de los ANOVAs de $2 \times 2$ se indican en las Tablas 2 (para los errores no forzados) y 3 (para los puntos conseguidos). El efecto de la autoestima resultó ser estadísticamente significativo para ambas variables del rendimiento (errores forzados y puntos conseguidos); la interacción entre la autoestima y la condición experimental fue significativa únicamente para la variable de errores no forzados. Los efectos globales de la autoestima tienden a indicar que ésta facilita la ocurrencia de errores no forzados (Tabla 2), así como también los puntos conseguidos (Tabla 3). Es decir, por una parte parece que interfiere en el rendimiento (facilitando los errores no forzados) y por otra lo mejora (incrementando los puntos conseguidos).
Figura 3. Representación gráfica de la interacción entre la autoestima y la presión deportiva, para la variable dependiente errores no forzados. SES = Self-Esteem Scale.

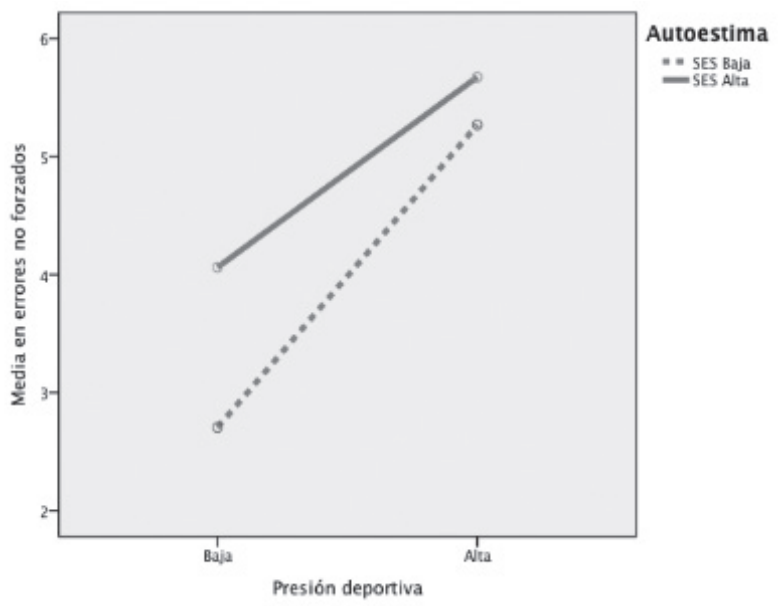

La interacción entre la autoestima y la condición experimental, observada para la variable de errores no forzados, sugiere un posible efecto modulador de la autoestima facilitando la ocurrencia de errores no forzados fundamentalmente cuando la presión deportiva es reducida (Figura 3). En contraste, encontramos un efecto general positivo de la autoestima sobre el rendimiento en términos de puntos conseguidos, aunque no un efecto modulador.

\section{Discusión}

En el presente estudio hemos tratado de probar dos hipótesis relacionadas con el efecto del miedo a la evaluación negativa sobre el rendimiento deportivo, y una hipótesis exploratoria sobre el posible efecto de la autoestima sobre el rendimiento deportivo. Mediante la primera hipótesis esperábamos que el miedo a la evaluación negativa actuara como modulador del efecto de la presión psicológica sobre el rendimiento, interfiriendo en éste en la condición de alta presión psicológica, y facilitándolo en la condición de baja presión psicológica. También hipotetizamos (segunda hipótesis) un incremento del rendimiento deportivo durante el periodo de alta presión deportiva en el grupo de participantes de bajo miedo. Finalmente, mediante la tercera hipótesis esperábamos que la autoestima facilitara el rendimiento deportivo, especialmente en la situación de alta presión deportiva. Los datos que hemos presentado sugieren que el miedo a la evaluación negativa constituye un factor de diferencias individuales de gran relevancia, que modula la influencia de la presión psicológica sobre el rendimiento deportivo. Así mismo, hemos obtenido evidencia que sugiere un posible papel de la autoestima, aunque los resultados son menos consistentes.

El principal resultado de nuestra investigación demuestra que el miedo a la evaluación negativa induce un doble 
efecto modulador sobre el rendimiento deportivo, según que el deporte se lleve a cabo en una condición de alta presión psicológica o en una condición de baja presión psicológica. Si el deporte se lleva a cabo en condiciones de alta presión psicológica, el miedo a la evaluación negativa interfiere de forma significativa con el rendimiento, lo cual se evidencia en ambas variables de rendimiento deportivo utilizadas (errores no forzados y puntos conseguidos). Este resultado confirma nuestra primera hipótesis, y es consistente con los datos obtenidos por Mesagno et al. (2012) con participantes jugadores de baloncesto. Así mismo, estos datos extienden los resultados obtenidos por Molina et al. (2014) sobre el efecto negativo de la sensibilidad a la ansiedad sobre el rendimiento deportivo en condiciones de alta presión.

En contraste, si el deporte se lleva a cabo en una condición de baja presión deportiva, el efecto del miedo a la evaluación negativa resulta ser facilitador del rendimiento deportivo. Este fenómeno ha sido constatado con la variable de puntos conseguidos (no con la variable de errores no forzados; aunque esta variable ha sido considerada como variable de rendimiento deportivo, en último término la variable "puntos conseguidos” refleja de forma más precisa el rendimiento del deportista). Tal resultado apoyaría también nuestra primera hipótesis, y sería coherente con la idea de que ciertos niveles de miedo pueden facilitar y motivar la conducta y mejorar el rendimiento (Arent y Landers, 2003; Chorot y Sandín, 1993).

Los resultados del presente estudio no apoyan nuestra segunda hipótesis, la cual consistía en una hipótesis formulada previamente por Mesagno et al. (2012), según la cual el bajo miedo a la evaluación negativa debería potenciar el rendimiento deportivo en las condiciones de alta presión psicológica. Ni en el estudio de Mesagno et al. ni en la presente investigación se proporciona evidencia que valide esta hipótesis. Tal hipótesis la fundamentaban los autores en que las situaciones de presión deberían incrementar moderadamente la "autoconciencia pública" (debido a que la atención de los espectadores se focaliza en el "ser público" del deportista) en los individuos con bajos niveles de temor a la evaluación negativa. Esto se asociaría a niveles moderados de ansiedad y auto-conciencia que, en último término facilitaría el rendimiento.

Los datos que hemos obtenido en relación con la autoestima, relativos a la principal variable del rendimiento deportivo, i.e., los puntos conseguidos, apoyan parcialmente nuestra tercera hipótesis, ya que esta variable de personalidad tiende a facilitar el rendimiento deportivo. Sin embargo, no encontramos un efecto modulador asociado a esta variable, constatándose que la autoestima favorece el rendimiento deportivo por igual en ambas condiciones de presión (la autoestima actúa sobre el rendimiento independientemente de las condiciones experimentales). Este efecto general de la autoestima favorecedor del rendimiento deportivo podría interpretarse en tér- minos de una posible mediación de variables positivas como la autoconfianza, tal y como se ha sugerido también para la relación entre la autoestima y otras formas de rendimiento (Rosli et al., 2011). Estos resultados confirman la evidencia presentada por otros autores que han sugerido una asociación positiva entre la autoestima y el rendimiento deportivo (Hines y Groves, 1989). A este respecto, Adie et al. (2008) defienden que los individuos con autoestima elevada tienden a percibir la competición deportiva como un reto, con más exigencia, que mejoraría su rendimiento deportivo; mientras que los participantes con autoestima baja perciben el deporte como una situación más amenazante, que perturbaría su rendimiento deportivo.

No obstante, nos ha llamado la atención el hecho de que el efecto significativo de la autoestima para la variable dependiente de errores no forzados denotase un efecto de reducción del rendimiento al incrementar el número de errores no forzados (la autoestima favorecía la ocurrencia de errores especialmente durante la condición de baja presión). Estos resultados irían en principio en contra de nuestra tercera hipótesis, en la que postulábamos que la autoestima siempre debería facilitar el rendimiento deportivo. Sin embargo, aparte de que la puntuación en errores no forzados reflejan menos fielmente el rendimiento deportivo que la variable de puntos conseguidos, el hecho de que los jugadores altos en autoestima cometan más errores no forzados durante la condición de baja presión podría deberse a que estos jugadores, por poseer mayor grado de autoconfianza, participan más y arriesgan más en el juego. Tal fenómeno sería consistente con la hipótesis defendida por Campbell (1990) sobre la asociación entre grado de participación, arriesgar en el juego, y autoconfianza. Tal vez esto explique que los participantes con elevada autoestima hayan cometido más errores no forzados que los individuos bajos en autoestima, indicando una mayor participación, asumiendo más riesgos y cometiendo más aciertos (golpes ganadores y puntos conseguidos), pero también más errores, al percibir la situación más como un reto que como una amenaza. En este sentido, en futuros estudios debería corregirse el número de errores según el grado de participación.

\section{Aplicaciones prácticas}

Mediante el presente estudio proporcionamos nueva evidencia sobre la implicación del miedo a la evaluación negativa y la autoestima en la práctica del deporte de competición en el ámbito educativo, y ampliamos la evidencia presentada recientemente por Molina et al. (2014) sobre los efectos de la sensibilidad a la ansiedad en el rendimiento deportivo. Tanto el miedo a la evaluación negativa como la sensibilidad a la ansiedad parecen influir de forma significativa en el rendimiento deportivo y deberían ser tenidas en cuenta si se desea maximizar dicho rendimiento. Debemos señalar, no obstante, 
que el tipo de diseño utilizado, así como también el tipo de participantes (una muestra de estudiantes adolescentes de enseñanza secundaria), no nos permite generalizar estos resultados al deporte de alta competición y a los deportistas profesionales. Para ello se precisa llevar a cabo nuevos estudios, con diseños apropiados, en el contexto de este tipo de población de deportistas. No obstante, merece la pena señalar que nuestros resultados apoyan en gran medida los datos referidos por Mesagno et al. (2012) sobre los efectos que tiene el miedo a la evaluación negativa en el rendimiento deportivo constatado en jugadores profesionales de baloncesto. Por tanto, basándonos en los datos referidos por Mesagno et al. (2012) y en los hallazgos obtenidos en el presente estudio, cabría afirmar que los efectos del miedo a la evaluación negativa sobre el rendimiento deportivo parecen ser independientes del tipo de competición de que se trate, ya que parecen ser similares en relación con el rendimiento deportivo de alta competición (i.e., situaciones deportivas de élite y/o profesionales) y con el rendimiento basado en competiciones de tipo educativo (i.e., situaciones escolares de competición deportiva).

\section{Referencias}

1. Adie, J. W., Duda, J. L. y Ntoumanis, N. (2008). Achievement goals, competition appraisals, and the psychological and emotional welfare of sport participants. Journal of Sport y Exercise Psychology, 30, 302-322.

2. Arent, S. M. y Landers, D. M. (2003). Arousal, anxiety, and performance: A reexamination of the inverted-U hypothesis. Research Quarterly for Exercise and Sport, 74, 436-444.

3. Aryana, M. (2010). Relationship between self-esteem and academic achievement amongst pre-universitysStudents, Journal of Applied Sciences, 10, 2474-2477.

4. Auweele, Y. V., De-Cuyper, B., Van-Mele, V. y Rzewnicky, R. (1993). Elite performance and personality: From description and prediction to diagnosis and Intervention. En R. N. Singer, M. Murphey, y L. K. Tennant (eds.), Handbook of Research on Sport Psychology (pp. 257-299). New York: Macmillan.

5. Baumeister, R. F. (1984). Choking under pressure: Self-consciousness and paradoxical effects of incentives on skillful performance. Journal of Personality and Social Psychology, 46, 610-620.

6. Baumeister, R. F. (1993). Self-esteem: The puzzle of low self-regard. New York: Plenum.

7. Beilock, S. L. y Carr, T. H. (2001). On the fragility of skilled performance: What governs choking under pressure? Journal of Experimental Psychology: General, 130, 701-725.

8. Buceta, J. M. (1996). Psicología y lesiones deportivas: prevención y recuperación. Madrid: Dykinson.

9. Campbell, J. D. (1990). Self-esteem and clarity of the self-concept. Journal of Personality and Social Psychology, 59, 538-549.

10. Coudevylle, G. R., Gernigon, C. y Martin, K. A. (2011). Self-esteem, self-confidence, anxiety and claimed self-handicapping: A mediational analysis. Psychology of Sport and Exercise, 12, 670-675.

11. Chorot, P. y Sandín, B. (1993). Effects of UCS intensity and duration of exposure of nonreinforced CS on conditioned electrodermal responses: An experimental analysis of the incubation theory of anxiety. Psychological Reports, 73, 931-941.

12. Gallego, M. J., Botella, C., Quero, S., Baños, R. M. y García-Palacios, A. (2007). Propiedades psicométricas de la escala de miedo a la evaluación negativa versión breve (BFNE) en muestra clínica. Revista de Psicopatología y Psicología Clínica, 12, 163-176.

13. Gimeno, F. y Ezquerro, M. (2006). Intervención psicológica en un caso de evitación interoceptiva en el deporte. Revista de Psicopatología y Psicología Clínica, 11, 99-106.

14. Hall, E. (2002). Defining choking: A qualitative examination. Unpublished Masters Thesis, Victoria University, Victoria, Australia.

15. Hammermeister, J. y Burton, D. (2001). Stress, appraisal, and coping revisited: Examining the antecedents of competitive state anxiety with endurance athletes. The Sport Psychologist, 15, 66-90.

16. Hill, D. M. y Shaw, G. (2013). A qualitative examination of choking under pressure in team sport. Psychology of Sport and Exercise, 14, 103-110.
17. Hill, D. M., Hanton, S., Matthews, N. y Fleming, S. (2010). Choking in sport: a review. International Review of Sport and Exercise Psychology, 3, 24-39.

18. Hines, S. y Groves, D. L. (1989). Sports competition and its influence on self-esteem development. Adolescence, 24, 861-869.

19. Jones, G. (1995). More than just a game: Research developments and issues in competitive anxiety in sport. British Journal of Psychology, 86, 449-478.

20. Kerr, J. H. (1985). The experience of arousal: A new basis for studying arousal effects in sport. Journal of Sport Sciences, 3, 169-179.

21. Leary, M. R. (1983). A brief version of the fear of negative evaluation scale. Personality and Social Psychology Bulletin, 9, 371-375.

22. Leary, M. R. (1992). Self-presentational processes in exercise and sport. Journal of Sport y Exercise Psychology, 14, 339-351.

23. Mesagno, C., Harvey, J. T. y Janelle, C. M. (2012). Choking under pressure: The role of fear of negative evaluation. Psychology of Sport and Exercise, 13, 60-68.

24. Mesagno, C., Marchant, D. y Morris, T. (2008). A pre-performance routine to alleviate choking in "choking-susceptible" athletes. The Sport Psychologist, 22, 439-457.

25. Molina, J., Sandín, B. y Chorot, P. (2014). Sensibilidad a la ansiedad y presión psicológica. Cuadernos de Psicología del Deporte, 14, 45-54.

26. Montero, P., Rueda, B. y Bermúdez, J. (2012). Relación de la personalidad tipo D y el agotamiento vital con las emociones negativas y el ajuste psicológico a la enfermedad cardíaca. Revista de Psicopatología y Psicología Clínica, 17, 93-106.

27. Rosenberg, M. (1965). Society and the adolescent self-image. Princeton, NJ: Princeton University Press.

28. Rosli, Y., Othman, H., Ishak, I., Lubis, S. H., Saat, N. Z. M. y Omar, B. (2011). Self-esteem and academic performance relationship amongst the second year undergraduate students of Universiti Kebangsaan Malaysia, Kuala Lumpur Campus. Procedia-Social and Behavioral Sciences, 60, 582-589.

29. Sandín, B. (2005). Evitación interoceptiva: Nuevo constructo en el campo de los trastornos de ansiedad. Revista de Psicopatología y Psicologia Clínica, 10, 103-114.

30. Sandín, B. (2008). El estrés psicosocial. Conceptos y consecuencias clínicas (2a ed.). Madrid: Klinik.

31. Sandín, B. (2010). Ejercicio físico y salud. Madrid: Klinik.

32. Sandín, B. y Chorot, P. (1989). The incubation theory of fear/anxiety: Experimental investigation in a human laboratory model of Pavlovian conditioning. Behaviour Research and Therapy, 27, 9-18.

33. Sandín, B., Chorot, P., Lostao, L. y Valiente, R.M. (2012). Screening del cáncer de mama: Afectación psicológica. Madrid: UNED.

34. Sandín, B., Chorot, P., Santed, M. A. y Valiente, R. M. (2002). Análisis factorial confirmatorio del Índice de Sensibilidad a la Ansiedad para Niños. Psicothema, 14, 333-339. 
35. Sandín, B., Chorot, P., Valiente, R. M., Santed, M.A. y Sánchez-Arribas, C. (1999). Estructura factorial de la Escala de Ansiedad Social para Niños-Revisada (SASC-R). Revista de Psicopatología y Psicología Clínica, 4, 103-113.

36. Sandín, B., Valiente, R. M., Chorot, P. y Santed, M. A (2005). Propiedades psicométricas del Índice de Sensibilidad a la Ansiedad. Psicothe$m a, 17,478-483$.
37. Watson, D. y Friend, R. (1969). Measurement of social-evaluative anxiety. Journal of Consulting and Clinical Psychology, 33, 448-457.

38. Woodman, T. y Hardy, L. (2001). Stress and anxiety. En R. N. Singer, H. A. Hausenblas y M. Janelle (eds.), Handbook of sport psychology (2a ed.) (pp. 290-318). New York: John Wiley and Sons, Inc. 